\title{
The effect of boron diffusions on the defect density and recombination at the (111) silicon-silicon oxide interface
}

\author{
H. Jin, ${ }^{1, a)}$ W. E. Jellett, ${ }^{1}$ Z. Chun, ${ }^{1}$ K. J. Weber, ${ }^{1}$ A. W. Blakers,${ }^{1}$ and P. J. Smith ${ }^{2}$ \\ ${ }^{1}$ Centre for Sustainable Energy Systems, Faculty of Engineering and Information Technology, The Australian \\ National University, Canberra, Austrialian Capital Territory 0200, Australia \\ ${ }^{2}$ Department of Chemistry, Faculty of Science, The Australian National University, Canberra, Australian \\ Capital Territory 0200, Australia
}

(Received 18 February 2008; accepted 7 March 2008; published online 26 March 2008)

\begin{abstract}
A combination of electron paramagnetic resonance (EPR) and minority carrier lifetime measurements is used to unambiguously demonstrate that the presence of a B diffusion layer at the surface of oxidized $\mathrm{Si}$ (111) wafers causes a significant increase in the interface defect density as well as interface recombination, compared to undiffused surfaces. EPR measurements show a nearly three-fold increase in the $\mathrm{Pb}$ center density, while the lifetime measurements indicate an increase in surface recombination activity by a factor of more than two, for B diffused samples with a sheet resistance of $\sim 250 \Omega / \square \square$. (C) 2008 American Institute of Physics. [DOI: 10.1063/1.2903698]
\end{abstract}

Shallow, heavily doped regions play an important role in commercial silicon solar cells, as they are used both for the formation of the $p-n$ junction as well as for high-low junctions in order to reduce recombination at the surfaces. The heavily doped region is most often formed through the diffusion of a suitable impurity (phosphorus or boron). The importance of these regions necessitates a good understanding of their properties. This includes the effect of the introduction of dopant atoms on the density and properties of defects at the interface of the silicon and the dielectric layer (such as $\mathrm{SiO}_{2}$ or PECVD $\mathrm{SiN}_{x}$ ) used to passivate the silicon surface. The $\mathrm{Si}-\mathrm{SiO}_{2}$ interface in particular is of considerable interest due to the excellent surface passivation that it affords.

The investigation of heavily doped silicon interfaces and, in particular, the determination of interface defect densities and associated capture cross sections, is more difficult than for the case of moderately doped material since capacitance-voltage $(C-V)$ measurements-the chief characterization technique-only yield limited information for highly doped material and with a greater error margin. In what appears to be the only study of its kind, the effect of B and $\mathrm{P}$ diffusions on this interface was investigated by Snel, ${ }^{1}$ using $C-V$ measurements at $77 \mathrm{~K}$. He concluded that both types of surface doping lead to an approximately linear increase in interface defect density with surface doping above a certain doping concentration, with the onset occurring at a lower dopant concentration for B than P doped surfaces. However, the results of this study were never replicated. Further, the values obtained for the interface defect density $D_{\text {it }}$ for moderately doped silicon are inconsistent with the values from the recent studies. ${ }^{2}$

In this paper, we investigate and compare the $\mathrm{Si}-\mathrm{SiO}_{2}$ interface of B diffused and undiffused, thermally oxidized, (111) oriented samples using electron paramagnetic resonance (EPR) measurements, and minority carrier lifetime measurements on metal-oxide semiconductor (MOS) structures as a function of applied voltage. ${ }^{3}$ The use of lifetime measurements on MOS structures at a sufficiently large applied bias voltage allows direct comparison of the emitter saturation current $J_{\text {oe }}$ of diffused and undiffused samples. For undiffused samples, a thin accumulation or inversion layer is created in the Si surface region when sufficient voltage bias is applied, making the measurement of $J_{\mathrm{oe}}$ values possible. The lifetime-voltage technique has the advantages over the more commonly used corona charging method of eliminating the possibility of corona introduced interface damage ${ }^{4,5}$ and surface potential fluctuations, ${ }^{6}$ which can render the interpretation of results on corona charged samples extremely difficult. EPR measurements allow direct determination of the density of unpassivated $P_{b}$ centers, the chief defect on (111) oriented $\mathrm{Si}-\mathrm{SiO}_{2}$ interfaces.

Boron diffusions were carried out using a liquid boron tribromide source in a diffusion furnace at a temperature of $900{ }^{\circ} \mathrm{C}$ to obtain a sheet resistance of around $250 \Omega / \square$, with variation across the sample of less than 5\%. Figure 1 shows the boron profile resulting from this diffusion.

Samples used for EPR measurements were $n$ type, $\sim 4000 \Omega \mathrm{cm}$, (111) $\mathrm{Cz}$ silicon wafers. Samples were cut with a diamond saw into $25 \mathrm{~mm} \times 2.5 \mathrm{~mm}^{2}$ pieces. They were subsequently etched to remove saw damage from the surfaces. Samples for lifetime-voltage measurements were

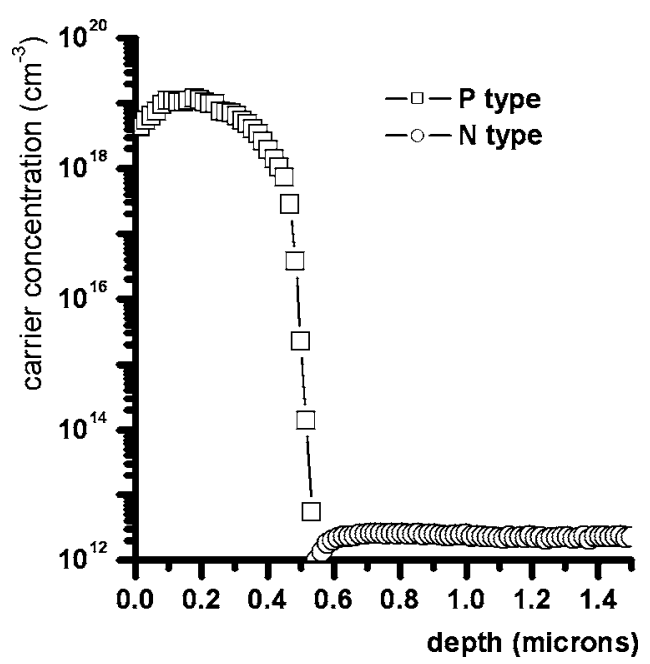

${ }^{a)}$ Electronic mail: hao.jin@anu.edu.au.

FIG. 1. The boron profile of the samples used for measurements. 
float zoned, $85 \Omega \mathrm{cm} n$ type, (111) oriented wafers.

After a standard RCA clean, a B diffusion was performed on both sides of selected samples. An oxide layer around $50 \mathrm{~nm}$ thick was then thermally grown on both sides at $1000{ }^{\circ} \mathrm{C}$ on all samples. A rapid thermal anneal was carried out on samples for EPR measurement at $700{ }^{\circ} \mathrm{C}$ for $3 \mathrm{~min}$ in a high flow of nitrogen gas to dehydrogenate the $P_{b}$ centers at the $\mathrm{Si}-\mathrm{SiO}_{2}$ interface.,

EPR measurements were undertaken using a Bruker $300 \mathrm{E}$ spectrometer operating at $x$ band $(9.44 \mathrm{GHz})$, fitted with an Oxford ES-9 liquid helium cryostat with temperature control via an Oxford ITC-4 controller. Measurements were done using a modulation frequency of $100 \mathrm{kHz}$, modulation amplitude of $1 \mathrm{G}$ and a microwave power of $20 \mu \mathrm{W}$ at a temperature of $7 \mathrm{~K} .20 \mu \mathrm{W}$ was observed to be nonsaturating. Samples were placed in a $3 \mathrm{~mm}$ internal diameter quartz EPR tubes, which were flushed with pure argon to remove oxygen. The sample tubes were sealed with rubber septa and the sample end frozen to $77 \mathrm{~K}$. The angle between the sample surface and the magnetic field is within an error of $3^{\circ}$. The $P_{b}$ center concentration was calculated by double integration of the original signal and comparison with a standard solution signal, obtained under similar conditions.

The experimental details for lifetime-voltage measurements are described in detail elsewhere. ${ }^{3}$ Measurements were carried out using the inductively coupled photoconductivity decay technique. ${ }^{10,11}$ Under conditions where the wafer bulk is in high level injection and the surfaces are in low level injection, the instantaneous decay time $\tau_{\text {inst }}$ is given by ${ }^{11}$

$$
1 / \tau_{\text {inst }}=1 / \tau_{\text {bulk, hli }}+\left(2 J_{\mathrm{oe}} n\right) /\left(q W n_{1}^{2}\right),
$$

where $\tau_{\text {bulk,hli }}$ is the high level injection lifetime of the wafer bulk, $n$ is the photogenerated excess carrier density in the wafer bulk, $W$ is the wafer thickness, $n_{i}$ is the intrinsic carrier concentration, $J_{\mathrm{oe}}$ is the emitter dark saturation current density, and $q$ the electronic charge. The value of $J_{\mathrm{oe}}$ can be determined from the slope of $1 / \tau_{\text {inst }}$ versus $n$, after Auger and radiative recombination have been accounted for. Both $\tau_{\text {inst }}$ and $J_{\text {oe }}$ were measured at an injection level of 4 $\times 10^{15} \mathrm{~cm}^{-3}$. For all measurements reported here, $n$ is uni-

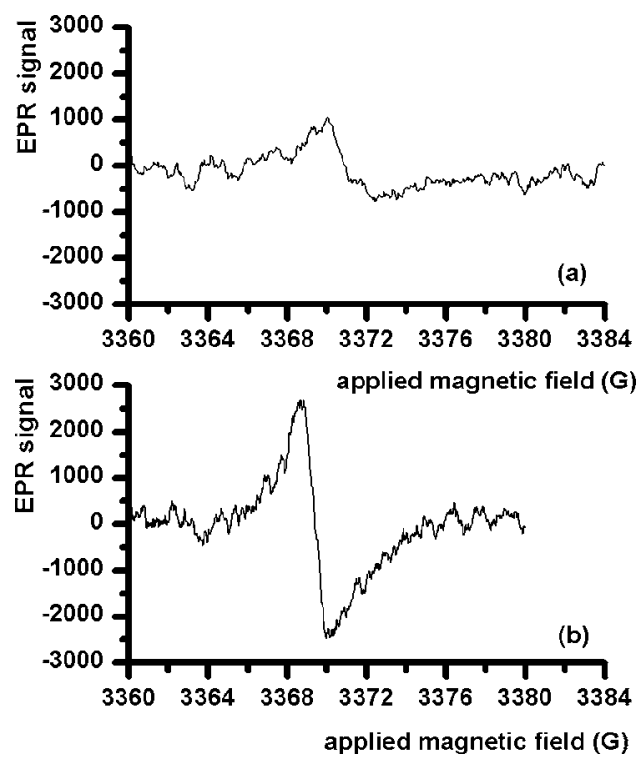

FIG. 2. EPR signals for undiffused sample (a) and boron diffused sample

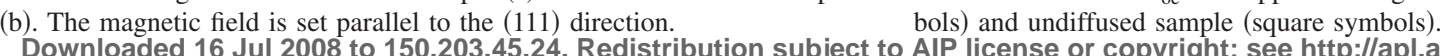

FIG. 3. Variation of $J_{\mathrm{oe}}$ with applied voltage for the diffused (triangle symbols) and undiffused sample (square symbols).

form throughout the wafer due to the high bulk lifetime and low surface recombination rates. Equation (1) is valid for both diffused and undiffused samples provided the surface remains in low level injection during the measurement. For the undiffused samples used here, this condition is met for $V_{\text {app }}<-4 \mathrm{~V}$ and $V_{\text {app }}>1 \mathrm{~V}$. For the diffused samples, the condition is satisfied for all (negative) bias voltages.

Figure 2 compares the EPR spectra for the undiffused (a) and $\mathrm{B}$ diffused (b) samples, while Table I compares the EPR parameters for the two samples. The values in Table I show that the EPR signal of the B diffused sample is a characteristic of the $P_{b}$ center, but the $P_{b}$ center density is substantially greater than that of the undiffused sample.

Figure 3 shows $J_{\text {oe }}$ as a function of applied voltage $V_{\text {app }}$ for both diffused and undiffused samples. The diffused sample was only measured in accumulation (negative bias), while the undiffused samples were measured with both positive and negative biases.

For the undiffused sample, the values of $J_{\mathrm{oe}}$ for large negative and positive bias voltages (corresponding to deep inversion and accumulation, respectively) are similar. This result also holds for (100) surfaces. ${ }^{3}$ This implies that the $\mathrm{Si}-\mathrm{SiO}_{2}$ (111) interface with heavy $n$ type or $p$ type surface doping can, in principle, be passivated equally well, if it could be ensured that the heavy doping does not alter the interface. This strongly suggests that the commonly observed poorer interface passivation of $p$-type emitters (compared to comparable $n$-type emitters) is the result of interface modification due to the heavy doping, rather than resulting from

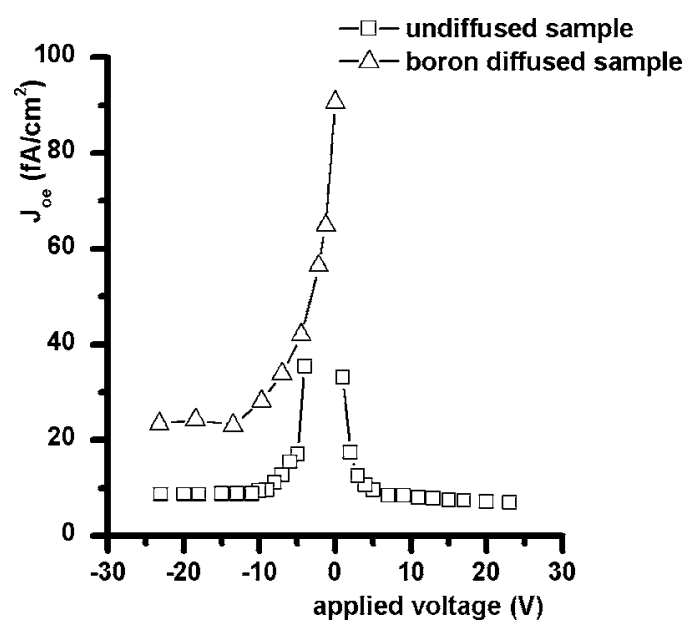


the inherent properties of the defects at the undiffused interface, such as a high electron to hole capture cross section $\left(\sigma_{n} / \sigma_{p}\right)$ ratio. $^{12}$

The diffused sample displays a substantially higher $J_{\mathrm{oe}}$ in accumulation $\left(24 \mathrm{fA} / \mathrm{cm}^{2}\right)$ than the undiffused sample in inversion $\left(9 \mathrm{fA} / \mathrm{cm}^{2}\right)$. Part of this increase is due to the presence of the emitter. However, the shallow emitter is expected to only contribute $\sim 3 \mathrm{fA} / \mathrm{cm}^{2}$ to $J_{\mathrm{oe}}$. The surface contribution to $J_{\mathrm{oe}}$ is therefore estimated to be $J_{\mathrm{oe}, \text { surf }}=21 \mathrm{fA} / \mathrm{cm}^{2}$. The increase in $J_{\text {oe,surf }}$ can only be attributed to an increase in interface recombination, resulting from an increase in the interface defect density or their capture cross sections.

It is important to note that $J_{\text {oe }}$ measurements were carried out on passivated (hydrogenated) samples, while EPR measurements were done on dehydrogenated samples in order to obtain the largest possible $P_{b}$ signal and, hence, maximize measurement accuracy. The results of the two measurements must therefore be compared with caution. The relative increase in $P_{b}$ density would be expected to translate to the same relative increase in (unpassivated) $P_{b}$ center density following hydrogenation only if the properties of the centers are completely unchanged. The EPR signature for diffused and undiffused samples is indeed almost identical. The relative increases in $J_{\mathrm{oe} \text {,surf }}$ (factor of 2.3) and $P_{b}$ centre density (factor of 2.7) are equal within the error of the measurements and we tentatively conclude that the increase in interface recombination on hydrogenated samples is chiefly due to an increase in unpassivated $P_{b}$ center density.

We also note that while our results are in qualitative agreement with those of Snel, ${ }^{1}$ the increase in interface defect density measured by Snel for B diffused surfaces of the same surface B concentration (greater than an order of magnitude) is far greater than reported here. Thus, while some interface degradation as a result of boron diffusions is probably unavoidable, our results show that, with careful processing, the extent of the degradation can be limited to levels that are acceptable for most applications.

In summary, the results of EPR and minority carrier lifetime measurements unambiguously show that the presence of a $\mathrm{B}$ diffusion results in a significant increase in the interface defect $\left(P_{b}\right.$ center $)$ density at the (111) $\mathrm{Si}-\mathrm{SiO}_{2}$ interface and a concomitant increase in interface recombination.

The authors would like to thank Profesor A. Stesmans in Department of Physics, University of Leuven for many useful advices on EPR operation and discussion on signal analysis, Miss Fiona Beck from the same centre for preparing the EPR samples, and Dr. Ronald Pace in Department of Chemistry, ANU on discussing with the EPR operation.

${ }^{1}$ J. Snel, Solid-State Electron. 24, 135 (1981).

${ }^{2}$ A. G. Aberle, J. Schmidt, and R. Brendel, J. Appl. Phys. 79, 1491 (1996).

${ }^{3}$ W. E. Jellett and K. J. Weber, Appl. Phys. Lett. 90, 042104 (2007).

${ }^{4}$ H. Jin, K. J. Weber, N. C. Dang, and W. E. Jellett, Appl. Phys. Lett. 90, 262109 (2007).

${ }^{5}$ A. Stesmans and V. V. Afanas'ev, Appl. Phys. Lett. 82, 2835 (2003).

${ }^{6}$ S. W. Glunz, D. Biro, S. Rein, and W. Warta, J. Appl. Phys. 86, 683 (1999).

${ }^{7}$ A. Stesmans and G. Vangorp, Appl. Phys. Lett. 57, 2663 (1990).

${ }^{8}$ P. K. Hurley, A. Stesmans, V. V. Afanas'ev, B. J. O'Sullivan, and E. O’Callaghan, J. Appl. Phys. 93, 3971 (2003).

${ }^{9}$ P. K. Hurley, B. J. O'Sullivan, F. N. Cubaynes, P. A. Stolk, F. P. Widdershoven, and J. H. Das, J. Electrochem. Soc. 149, G194 (2002).

${ }^{10}$ E. Yablonovitch and T. J. Gmitter, Solid-State Electron. 35, 261 (1992).

${ }^{11}$ D. E. Kane and R. M. Swanson, Proceedings of the 18th Eighteenth IEEE Photovoltaic Specialists Conference, Las Vegas (IEEE, New York, 1985), p. 578.

${ }^{12}$ A. G. Aberle, S. W. Glunz, and W. Warta, J. Appl. Phys. 71, 4422 (1992). 